\title{
Tonic Suppression of the Mesolimbic Dopaminergic System by Enhanced Corticotropin-Releasing Factor Signaling Within the Bed Nucleus of the Stria Terminalis in Chronic Pain Model Rats
}

\author{
Daiki Takahashi, ${ }^{\star}$ Yuta Asaoka, ${ }^{\star}$ Keisuke Kimura, ${ }^{*}$ Ryuto Hara, Saya Arakaki, Keisuke Sakasai, Hiroe Suzuki, \\ Naoki Yamauchi, Hiroshi Nomura, Taiju Amano, and Masabumi Minami \\ Department of Pharmacology, Graduate School of Pharmaceutical Sciences, Hokkaido University, Sapporo 060-0812, Japan
}

Although dysfunction of the mesolimbic dopaminergic system has been implicated in chronic pain, the underlying mechanisms remain to be elucidated. We hypothesized that increased inhibitory inputs to the neuronal pathway from the dorsolateral bed nucleus of the stria terminalis (dIBNST) to the ventral tegmental area (VTA) during chronic pain may induce tonic suppression of the mesolimbic dopaminergic system. To test this hypothesis, male Sprague Dawley rats were subjected to spinal nerve ligation to induce neuropathic pain and then spontaneous IPSCs (sIPSCs) were measured in this neuronal pathway. Whole-cell patch-clamp electrophysiology of brain slices containing the dIBNST revealed that the frequency of sIPSCs significantly increased in VTA-projecting dlBNST neurons 4 weeks after surgery. Next, the role of corticotropin-releasing factor (CRF) signaling within the dIBNST in the increased sIPSCs was examined. CRF increased the frequency of sIPSCs in VTA-projecting dlBNST neurons in sham-operated controls, but not in chronic pain rats. By contrast, NBI27914, a CRF type 1 receptor antagonist, decreased the frequency of sIPSCs in VTA-projecting dlBNST neurons in the chronic pain rats, but not in the control animals. In addition, histological analyses revealed the increased expression of CRF mRNA in the dIBNST. Finally, bilateral injections of NBI27914 into the dlBNST of chronic pain rats activated mesolimbic dopaminergic neurons and induced conditioned place preference. Together, these results suggest that the mesolimbic dopaminergic system is tonically suppressed during chronic pain by enhanced CRF signaling within the dIBNST via increased inhibitory inputs to VTA-projecting dlBNST neurons.

Key words: bed nucleus of the stria terminalis; corticotropin-releasing factor; dopamine; extended amygdala; pain; ventral tegmental area

Significance Statement

The comorbidity of chronic pain and depression has long been recognized. Although dysfunction of the mesolimbic dopaminergic system has been implicated in both chronic pain and depression, the underlying mechanisms remain to be elucidated. Here, we show that the inhibitory inputs to the neuronal pathway from the dorsolateral bed nucleus of the stria terminalis (dIBNST) to the ventral tegmental area increase during chronic pain. This neuroplastic change is mediated by enhanced corticotropin-releasing factor signaling within the dlBNST that leads to tonic suppression of the mesolimbic dopaminergic system, which may be involved in the depressive mood and anhedonia under the chronic pain condition.

\section{Introduction}

The comorbidity of chronic pain and depression has long been recognized in the clinical setting (Bair et al., 2003; Doan et al.,
2015), and several preclinical studies have reported depressionlike behaviors in animal models of chronic pain (Yalcin et al., 2014). These findings suggest a common neuronal basis for 
chronic pain and depression. The mesolimbic dopaminergic pathway from the ventral tegmental area (VTA) to the nucleus accumbens (NAc) is a key player in the mesolimbic reward circuit, and dysfunction in this pathway has been implicated in depression (Nestler et al., 2006; Russo et al., 2013; Minami et al., 2017). Dysfunction of the mesolimbic dopaminergic pathway has also been implicated in chronic pain. Dopamine release in the NAc induced by reward drugs such as morphine and cocaine is suppressed in animal models of neuropathic pain (Ozaki et al., 2002; Taylor et al., 2015). Furthermore, we previously reported that intra-NAc dopamine release induced by sucrose solution intake as a reward is suppressed in chronic pain rats (Kato et al., 2016). These findings suggest that suppression of dopaminergic neurotransmission in the mesolimbic reward circuit may be a common neuroplastic change underlying chronic pain and depression.

The bed nucleus of the stria terminalis (BNST), together with the central amygdala (CeA), form the extended amygdala (Alheid and Heimer, 1988), which is implicated in the regulation of negative emotional states, such as fear, anxiety, and aversion (Davis et al., 2010; Minami, 2019). Optogenetic studies have demonstrated that photoactivating the BNST neuron terminals projecting into the VTA induces reward-related behaviors in a real-time place preference test (Jennings et al., 2013; Kim et al., 2013), suggesting that VTA-projecting BNST neurons positively regulate the mesolimbic reward circuit. In the present study, we hypothesized that increased inhibitory inputs to the VTAprojecting BNST neurons during chronic pain may induce tonic suppression of the mesolimbic dopaminergic system. To test this hypothesis, we first examined spontaneous IPSCs (sIPSCs) in the VTA-projecting dorsolateral BNST (dlBNST) neurons of brain slices prepared from chronic pain rats. Whole-cell patch-clamp recordings revealed that the frequency of sIPSCs in the VTAprojecting dlBNST neurons increased in the chronic pain group compared with those in the sham-operated control group. Next, we examined the involvement of corticotropin-releasing factor (CRF) signaling within the dlBNST in the increased sIPSCs during chronic pain, because we previously demonstrated that CRF signaling within the dlBNST plays an important role in the aversive response to acute pain (Ide et al., 2013). Bath application of CRF increased the frequency of sIPSCs in the VTA-projecting neurons of the sham-operated control, but not the chronic pain rats. By contrast, bath application of the CRF type 1 receptor antagonist NBI27914 decreased the frequency of sIPSCs in the VTA-projecting neurons of the chronic pain, but not the shamoperated group. These results suggest tonic inhibition of the VTA-projecting dlBNST neurons by enhanced CRF signaling during chronic pain. Finally, we revealed that bilateral injections of NBI27914 into the dlBNST of chronic pain rats increased the intra-VTA c-fos mRNA expression and the intra-NAc dopamine release, and induced conditioned place preference (CPP). These findings suggest that the mesolimbic dopaminergic system is tonically suppressed during chronic pain by enhanced CRF signaling within the dlBNST via increased inhibitory inputs to VTAprojecting dlBNST neurons.

\section{Materials and Methods}

Animals. In total, 219 male Sprague Dawley rats (Japan SLC) weighing $240-310 \mathrm{~g}$ were used. The animals were housed in a room with a constant ambient temperature $\left(23 \pm 1^{\circ} \mathrm{C}\right)$ under a $12 \mathrm{~h}$ light/dark cycle, with food and water available ad libitum. All experiments were performed with approval of the Hokkaido University Institutional Animal Care and Use Committee.
Drugs. CRF was from the Peptide Institute. NBI27914 hydrochloride (a selective CRF type 1 receptor antagonist) was from Tocris Bioscience. Kynurenic acid was from Sigma-Aldrich. The stock solution of CRF in the electrophysiological experiments was prepared at a concentration of $500 \mu \mathrm{M}$ in $\mathrm{H}_{2} \mathrm{O}$ containing $0.1 \%$ bovine serum albumin (BSA; SigmaAldrich). The stock solution of NBI27914 was prepared at a concentration of $2 \mathrm{~mm}$ in dimethylsulfoxide (DMSO). Before the bath application, the NBI27914 stock solution was diluted to the final concentration with recording solution. The CRF stock solution was diluted to the final concentration with recording solution containing $0.1 \%$ BSA. NBI27914 was dissolved in DMSO and then diluted with saline for the behavioral and in vivo microdialysis experiments. The final concentrations of NBI27914 and DMSO were $1 \mathrm{nmol} / \mu$ land $4.2 \%$, respectively.

Surgical procedures and injections. Surgery was performed under anesthesia with isoflurane (2\%). Lidocaine (Aspen Japan) was topically administered at the incision sites to alleviate pain. Surgery was performed under pentobarbital anesthesia (50 mg/kg, i.p.) in some animals.

The neuropathic pain model rats were prepared by spinal nerve ligation (SNL) according to the method of Li et al. (2000) with some modifications. Briefly, under anesthesia, the left lumbar fifth (L5) spinal nerve was tightly ligated using a 6-0 silk suture and cut distal to the ligature. Sham-operated control rats underwent an identical surgical procedure, but the spinal nerves were not ligated or cut. To assess tactile allodynia, the von Frey test was conducted as described previously (Chaplan et al., 1994). The rats were individually confined in wire-mesh cages, and calibrated von Frey filaments $(0.4-15 \mathrm{~g})$ were applied to the plantar surface of the ipsilateral hindpaw following a habituation period of at least 30 $\min$. The $50 \%$ paw withdrawal threshold was determined using the updown method (Chaplan et al., 1994). The tests were conducted $1 \mathrm{~d}$ before and every $7 \mathrm{~d}$ after the surgery. Rats that showed motor impairment after surgery or did not show tactile allodynia were excluded from the following procedures. Twenty-one animals were excluded due to these exclusion criteria.

For electrophysiological experiments, retrograde tracer was injected into the VTA 3-7 d before the slice preparation. Specifically, the rats were fixed in a stereotaxic apparatus (SR-6R-HT; Narishige) under anesthesia, and an incision was made in the scalp. Small holes were drilled in the skull, and a 33-gauge Hamilton syringe connected to a microsyringe pump (SYS-MICRO4; World Precision Instruments) was inserted. The animals were unilaterally injected with $0.3-0.4 \mu \mathrm{l}$ of red or green retrobeads (Lumafluor) into the VTA $(-5.5 \mathrm{~mm}$ rostral, $1.0 \mathrm{~mm}$ lateral, $-9.0 \mathrm{~mm}$ ventral to bregma) (Paxinos and Watson, 2007) at a constant rate of $0.075 \mu \mathrm{l} / \mathrm{min}$ and left for an additional $5 \mathrm{~min}$ to prevent backflow. The injection site was checked during slice preparation. Eleven animals were excluded because the injection site was out of the VTA.

For in vivo microdialysis experiments, under anesthesia, 25-gauge stainless guide cannulae [outer diameter (o.d.), $0.5 \mathrm{~mm}$; inner diameter (i.d.), $0.22 \mathrm{~mm}$ ] for microinjection were implanted bilaterally above the dlBNST ( $-0.75 \mathrm{~mm}$ rostral, $1.6 \mathrm{~mm}$ lateral, $5.2 \mathrm{~mm}$ ventral to bregma) with a tilt of $30^{\circ}$ to the caudal side, and a microdialysis guide cannula (o.d., $0.5 \mathrm{~mm}$, AG-7; Eicom) was implanted unilaterally $1.0 \mathrm{~mm}$ above the NAc shell $(1.6 \mathrm{~mm}$ rostral, $0.9 \mathrm{~mm}$ lateral, $6.5 \mathrm{~mm}$ ventral to the bregma). Implantation of these guide cannulae was performed $24-27 \mathrm{~d}$ after the SNL surgery. After implantation, the animals were individually housed in cages for a recovery period of 3-6 d.

Rats for the behavioral experiments were implanted bilaterally with 25 -gauge stainless steel guide cannulae $1.5 \mathrm{~mm}$ above the dlBNST $(0.0$ $\mathrm{mm}$ rostral, $1.6 \mathrm{~mm}$ lateral, $5.0 \mathrm{~mm}$ ventral to bregma). After surgery, the rats were individually housed in cages for a recovery period of at least $6 \mathrm{~d}$, and handled for 1-2 min each day for 3 consecutive days before the behavioral experiments.

For the intra-dlBNST drug injections, 33-gauge stainless steel injection cannulae (o.d., $0.2 \mathrm{~mm}$; i.d., $0.08 \mathrm{~mm}$ ) were inserted bilaterally into the guide cannulae. The injection cannulae protruded $1.5 \mathrm{~mm}$ from the tip of the guide cannulae to reach the dlBNST. The injection cannulae were attached to a microinjection pump (CMA) via PE 8 polyethylene tubing. Drugs were administrated bilaterally in a volume of $0.5 \mu \mathrm{l} /$ side at a rate of $0.5 \mu \mathrm{l} / \mathrm{min}$, and the injection cannulae were left in place for an additional $1 \mathrm{~min}$ after the injections to prevent backflow. 
Slice preparation for electrophysiology. The rats were deeply anesthetized with sodium pentobarbital and transcardially perfused with ice-cold cutting solution containing the following (in mM): $92 \mathrm{~N}$-methylD-glucamine, $2.5 \mathrm{KCl}, 30 \mathrm{NaHCO}_{3}, 1.25 \mathrm{NaH}_{2} \mathrm{PO}_{4}, 25$ glucose, 5 ascorbic acid, $0.5 \mathrm{CaCl}_{2}, 20$ HEPES, $10 \mathrm{MgSO}_{4}$, 2 thiourea, 3 sodium pyruvate, and $12 \mathrm{~N}$-acetyl-L-cysteine, oxygenated with $95 \% \mathrm{O}_{2} / 5 \% \mathrm{CO}_{2}$ at $\mathrm{pH}$ $7.3 \pm 0.1$ adjusted with hydrochloric acid. The brain was quickly removed and coronal slices ( $250 \mu \mathrm{m}$ thick) containing the BNST were prepared in ice-cold cutting solution with a vibratome (VT1200S; Leica Microsystems). The slices were incubated for $15 \mathrm{~min}$ at $30-34^{\circ} \mathrm{C}$ in the cutting solution and subsequently kept in the recording solution containing the following (in mM): $119 \mathrm{NaCl}, 2.5 \mathrm{KCl}, 24 \mathrm{NaHCO}_{3}, 1.25$ $\mathrm{NaH}_{2} \mathrm{PO}_{4}, 12.5$ glucose, $2 \mathrm{CaCl}_{2}$, and $2 \mathrm{MgSO}_{4}$, oxygenated with $95 \%$ $\mathrm{O}_{2} / 5 \% \mathrm{CO}_{2}$ at room temperature for at least $1 \mathrm{~h}$. The slices were transferred to a submerged recording chamber on an upright microscope (BX50WI; Olympus) and constantly superfused with recording solution at $35 \pm 1{ }^{\circ} \mathrm{C}$ saturated with $95 \% \mathrm{O}_{2} / 5 \% \mathrm{CO}_{2}$ at a flow rate of $1 \mathrm{ml} / \mathrm{min}$. Glass pipettes were pulled from thin-walled borosilicate glass capillaries with a micropipette puller (Model P-1000IVF; Sutter Instruments). Tip resistance was 4.5-9.0 M $\Omega$.

Classification of dlBNST neurons. Because dlBNST neurons have been categorized into three distinct types (Hammack et al., 2007), we identified the cell types by assessing the membrane potential responses to hyperpolarizing and depolarizing current injections. Current-clamp recordings were conducted to characterize dlBNST neurons. Hyperpolarization-activated cation current $\left(I_{\mathrm{h}}\right)$ was identified as described previously (Yamauchi et al., 2018). Specifically, the occurrence of $I_{\mathrm{h}}$ was verified by the presence of a "voltage-sag" equal to or $>10 \%$ of the total voltage deflection upon the hyperpolarizing current injection, which elicited a membrane voltage of $\sim-100 \mathrm{mV}$. To monitor the responses of dlBNST neurons to hyperpolarizing current injections, the initial membrane potential was adjusted to $-60 \mathrm{mV}$, and a series of currents $(-40$ pA steps, $400 \mathrm{~ms}$ in duration) ranging from 0 to $-200 \mathrm{pA}$ was injected. To monitor the responses to depolarizing current injections, the initial membrane potential was adjusted to $-80 \mathrm{mV}$, and a series of currents (+40 pA steps, $400 \mathrm{~ms}$ in duration) ranging from 0 to $200 \mathrm{pA}$ was injected. $I_{\mathrm{h}}$-positive neurons that exhibited regular firing patterns in response to depolarizing current injections were classified as type $I, I_{\mathrm{h}}{ }^{-}$ positive neurons exhibiting rebound spiking in response to termination of hyperpolarizing current injections and burst spiking in response to depolarizing current injections were classified as type II, and $I_{\mathrm{h}}$-negative neurons were classified as type III.

Measurement of spontaneous IPSCs. VTA-projecting neurons labeled with retrobeads were visualized using epifluorescence and a $40 \times$ objective (LUMPlanLF N 40×/0.80; Olympus). Glass pipettes were filled with a high chloride internal solution containing the following (in $\mathrm{mm}$ ): 110 $\mathrm{KOH}, 2 \mathrm{MgCl}_{2}$, $50 \mathrm{KCl}, 0.2$ EGTA, $2 \mathrm{Na}_{2}$-ATP, $0.3 \mathrm{Na}_{3}$-GTP, 10 HEPES, and 0.1 spermine, adjusted to $\mathrm{pH} 7.3 \pm 0.1$ with gluconic acid. VTAprojecting dlBNST neurons were initially classified into the three types in current-clamp mode. After classification, the membrane potential was held at $-60 \mathrm{mV}$ under voltage-clamp mode and the sIPSCs were recorded. Kynurenic acid ( $2 \mathrm{~mm}$ ) was added to the recording solution to inhibit EPSCs. After observing a stable holding current for $3 \mathrm{~min}, 1 \mu \mathrm{M}$ CRF or $1 \mu \mathrm{M}$ NBI27914 was perfused for 2 or $15 \mathrm{~min}$, respectively. sIPSCs were analyzed during the $0-3 \mathrm{~min}$ before and $12-15 \mathrm{~min}$ after the start of the CRF or NBI27914 application. All data were acquired with a Multiclamp 700B amplifier and pClamp10 software (Molecular Devices). Data from the neurons whose resting membrane potential was more positive than $-50 \mathrm{mV}$ or in which the action potential did not overshoot were excluded from statistical analyses. Access resistance was monitored by injecting a current $(-5 \mathrm{mV}, 50 \mathrm{~ms})$ every $30 \mathrm{~s}$. Data from the neurons whose access resistance changed by $20 \%$ or more during recordings were excluded from statistical analyses. Data from 16 animals were excluded due to these exclusion criteria. The frequency and amplitude of the sIPSCs were analyzed using the Mini Analysis Program (Synaptosoft).

Measurement of firing rates. Firing rates were recorded in cell-attached mode for $3 \mathrm{~min}(0 \mathrm{mV}$, voltage-clamp mode). Glass pipettes were filled with an internal solution containing the following (in $\mathrm{mM}$ ): $150 \mathrm{KOH}, 2$ $\mathrm{MgCl}_{2}, 10 \mathrm{KCl}, 0.2$ EGTA, $2 \mathrm{Na}_{2}$-ATP, $0.3 \mathrm{Na}_{3}$-GTP, 10 HEPES, and 0.1 spermine, adjusted to $\mathrm{pH} 7.3 \pm 0.1$ with gluconic acid. To examine the effect of NBI27914 on firing rates, $1 \mu \mathrm{M}$ NBI27914 was perfused for 15 $\mathrm{min}$, and firing rates were analyzed during the $0-3$ min before and $12-15$ min after the start of the NBI27914 application. After recording the firing rates, the cell membrane was broken by negative pressure, and the neurons were classified into three types in current-clamp mode. Data from the neurons whose resting membrane potential was more positive than $-50 \mathrm{mV}$ or in which the action potential did not overshoot were excluded from statistical analyses. Data from 4 animals were excluded due to these exclusion criteria.

FISH and immunohistochemistry. After transcardial perfusion with 0.1 M sodium phosphate buffer (PB) followed by $4 \%$ paraformaldehyde (PFA) in $\mathrm{PB}$, the brains were immediately removed and immersed in the same fixative for $3-4 \mathrm{~d}$ at $4^{\circ} \mathrm{C}$. Subsequently, the PFA solution was replaced with $30 \%$ sucrose in $\mathrm{PB}$ and the tissue was left in this solution until it sank to the bottom. Then the fixed brains were embedded in frozen section compound and stored at $-80^{\circ} \mathrm{C}$ until use. Coronal sections $(40$ $\mu \mathrm{m})$ were prepared using a cryostat (CM3050S; Leica Microsystems).

FISH for CRF mRNA was performed as described previously (Kono et al., 2017). Briefly, a digoxigenin (DIG)-labeled cRNA probe was prepared using a cDNA fragment of CRF (GenBank accession no. BC119036) as a template for in vitro transcription. The sections were treated with the following incubation steps: acetylation with $0.25 \%$ acetic anhydride in $0.1 \mathrm{M}$ triethanolamine- $\mathrm{HCl}, \mathrm{pH} 8.0$, for $10 \mathrm{~min}$, washed with $2 \times$ saline-sodium citrate (SSC) and then briefly $0.1 \times$ SSC, and then prehybridization for $20 \mathrm{~min}$ in hybridization buffer (50\% formamide, 33 mм Tris- $\mathrm{HCl}, \mathrm{pH} 8.0,0.02 \%$ Ficoll, $0.02 \%$ polyvinylpyrrolidone, $0.02 \%$ bovine serum albumin, $0.6 \mathrm{M} \mathrm{NaCl}, 200 \mu \mathrm{g} / \mathrm{ml}$ tRNA, $1 \mathrm{~mm}$ EDTA, and $10 \%$ dextran sulfate). Hybridization was performed at $63.5^{\circ} \mathrm{C}$ for $14 \mathrm{~h}$ in hybridization buffer supplemented with a DIG-labeled cRNA probe. Posthybridization washing was successively performed at $61^{\circ} \mathrm{C}$ with $5 \times$ SSC for $30 \mathrm{~min}, 4 \times$ SSC containing $50 \%$ formamide for $30 \mathrm{~min}, 2 \times$ SSC containing $50 \%$ formamide for $30 \mathrm{~min}$, and then $0.1 \times$ SSC twice for 15 min each. Sections were rinsed with $0.1 \times$ SSC for 5 min at room temperature. The blocking procedure was performed by sequentially incubating the sections at room temperature in NTE buffer $(0.5 \mathrm{M} \mathrm{NaCl}, 10$ $\mathrm{mm}$ Tris-HCl,pH 8.0, and $5 \mathrm{~mm}$ EDTA) for $5 \mathrm{~min}, 20 \mathrm{~mm}$ iodoacetamide in NTE buffer for $20 \mathrm{~min}$, NTE buffer for $5 \mathrm{~min}$, TNT buffer $(0.1 \mathrm{M}$ Tris-HCl, pH 7.4, and $0.15 \mathrm{M} \mathrm{NaCl}$ ) for $5 \mathrm{~min}$, DIG blocking solution containing $1 \%$ blocking reagent (Roche Diagnostics) and 10\% normal sheep serum in TNT buffer for $30 \mathrm{~min}$, TNT buffer briefly, and 0.5\% tyramide signal amplification (TSA) blocking reagent (PerkinElmer) in TNT buffer for $30 \mathrm{~min}$. After a brief rinse with TNT buffer, the sections were incubated with peroxidase-conjugated anti-DIG antibody (1:1000; Roche Diagnostics) in DIG blocking solution for $1 \mathrm{~h}$. Then, after washing in TNT buffer twice for 5 min each, fluorescence detection was performed using a TSA plus Cy3 system (PerkinElmer). The sections were incubated with $\mathrm{Cy} 3$ amplification reagent in $1 \times$ plus amplification diluent for $10 \mathrm{~min}$ and then washed with TNT buffer three times for $5 \mathrm{~min}$ each. Throughout the procedures from prehybridization to the fluorescence detection of DIG, $0.0005-0.001 \%$ Tween 20 was added to the buffers to facilitate the access of the cRNA probe and the anti-DIG antibody to their targets. Following a rinse with PBS, the sections were mounted on glass slides using Vectashield hard set mounting medium with DAPI (Vector Laboratories).

To examine c-fos mRNA expression in the VTA after bilateral injections of NBI27914 $(0.5 \mathrm{nmol} / \mathrm{side})$ or vehicle into the dlBNST of SNL rats, the animals were transcardially perfused with PB followed by $4 \%$ PFA in PB at 30 min after the intra-dlBNST injections; the FISH procedure was similar to the above description. Briefly, a DIG-labeled cRNA probe for c-fos mRNA was prepared using a c-fos cDNA fragment (GenBank accession no. BC119036). In this case, FISH was followed by the immunohistochemical detection of tyrosine hydroxylase (TH). After washing in PBS containing $0.3 \%$ Triton X-100 (PBST) twice for $10 \mathrm{~min}$ each, the sections were incubated in blocking solution (5\% normal donkey serum in PBST) for $1 \mathrm{~h}$ and then with an anti-TH antibody (catalog \#MAB318, RRID:AB 2201528; Millipore) diluted with blocking solution (1:1000) overnight at $4^{\circ} \mathrm{C}$. After washing in PBST three times for $10 \mathrm{~min}$ each, the primary antibody was visualized with alexa647-labeled donkey 
anti-mouse IgG (1:500, catalog \#A31571, RRID:AB 162542; Life Technologies). After washing with PBS three times for $10 \mathrm{~min}$ each, the sections were mounted on glass slide using Vectashield hard set mounting medium with DAPI.

Image acquisition and cell counting. To analyze CRF mRNA expression, high-contrast bright field images were obtained using a fluorescence microscope (BZ-X710; Keyence) with a $4 \times$ objective lens (CFI Plan Apo $\lambda$ $4 \times / 0.2$; Nikon;). Using the high-contrast bright-field images, regionsof-interest (ROIs, $362 \times 273 \mu \mathrm{m}$ ) were placed on the dlBNST and CeA by an experimenter blinded to the fluorescent signals of CRF mRNA. Using a $40 \times$ objective lens (CFI Plan Apo $\lambda$ 40×/0.95; Nikon), fluorescent images for CRF mRNA (Cy3) and cell nuclei (DAPI) were obtained from each ROI. Then, using an optical sectioning algorithm (Keyence), the areas of analyses were $z$-sectioned in $0.6 \mu \mathrm{m}$ optical sections. The same scanning settings were used throughout the experiment to allow for comparisons across groups and all fluorescent images were analyzed using ImageJ software. Using the "Cell Counter" plugin in ImageJ software, relatively large nuclei stained with DAPI, which were presumed to be neuronal cells, were marked and then the number of marked cells was counted. Subsequently, the number of marked cells showing Cy3 signals (signals for CRF mRNA) was counted.

To analyze c-fos mRNA expression, TH fluorescent images were obtained using a fluorescence microscope with a $4 \times$ objective lens. Using the TH fluorescent images, ROIs $(362 \mu \mathrm{m} \times 273 \mu \mathrm{m})$ were placed on the VTA by an experimenter blind to fluorescent signals of c-fos mRNA and then the fluorescent images for c-fos mRNA (Cy3) and TH (Alexa Fluor 647) were obtained from each ROI with a $40 \times$ objective lens. Using an optical sectioning algorithm, the areas of analyses were $z$-sectioned in 0.6 $\mu \mathrm{m}$ optical sections; the same scanning settings were used throughout the experiment to allow for comparisons across groups. Using the "Cell Counter" plugin in ImageJ software, TH-positive and double-positive (TH- and c-fos mRNA-positive) cells were counted.

In vivo microdialysis. In vivo microdialysis was conducted in unanesthetized freely moving rats. A microdialysis probe (dialysis membrane: $1000 \mathrm{kDa}$ molecular weight cutoff polyethylene membrane, length 1.0 $\mathrm{mm}$; o.d., $0.22 \mathrm{~mm}$; A-I-7-01; Eicom) was inserted through the guide cannula and continuously perfused with Ringer's solution $(147 \mathrm{~mm}$ $\mathrm{NaCl}, 4 \mathrm{~mm} \mathrm{KCl}, 2.3 \mathrm{~mm} \mathrm{CaCl}_{2}$ ) at a flow rate of $1 \mu \mathrm{l} / \mathrm{min}$. The rats were placed in a Plexiglas chamber (width $\times$ length $\times$ height: $30 \times 30 \times 35$ $\mathrm{cm}$ ). After the extracellular dopamine level was stabilized, $35 \mathrm{~min}$ dialysate fractions were collected as baseline samples. After collecting the baseline samples, the animals received bilateral intra-dlBNST injections of NBI27914 (0.5 nmol/side), and then $185 \mathrm{~min}$ fractions were collected. Each dialysate sample was separated on a liquid chromatography column (Eicompak PP-ODS II, $4.6 \mathrm{~mm}$ i.d. $\times 30 \mathrm{~mm}$; Eicom) at $25^{\circ} \mathrm{C}$ using $0.1 \mathrm{M}$ phosphate buffer, pH 5.4, containing 500 or $600 \mathrm{mg} / \mathrm{L}$ sodium 1-decanesulfonate, $50 \mathrm{mg} / \mathrm{L}$ ethylene diamine tetraacetic acid, and $2 \%$ methanol at a constant flow rate of $0.5 \mathrm{ml} / \mathrm{min}$. Dopamine contents were measured using an electrochemical detector (HTEC-500; Eicom), with a working electrode set at $+400 \mathrm{mV}$ versus an $\mathrm{Ag} / \mathrm{AgCl}$ reference electrode. Chromatogram peaks were analyzed using the PowerChrom datarecording system (Eicom). Data are expressed as percentage of baseline, which was calculated as the average of the three baseline samples. Data from rats with a baseline value $<0.025 \mathrm{pg} / \mu \mathrm{l}$, a baseline value with a large variance (defined as a $>30 \%$ difference among the three baseline samples) or a bursting increase in serotonin level probably due to microhemorrhage were excluded from statistical analyses. Three animals were excluded due to these exclusion criteria.

Conditioned place preference (CPP) test. CPP tests were conducted as described previously (Shinohara et al., 2014). The CPP chamber consisted of two equal-sized compartments $(30 \times 30 \times 30 \mathrm{~cm})$ with distinct tactile and visual cues (one compartment had a black floor and walls with a stainless steel stripe-like grid on the floor, and the other had a white floor and walls with a stainless steel grid on the floor), which were separated by a removable partition (Muromachi Kikai). The CPP chambers were set in sound-attenuating boxes equipped with a ventilating fan. On days 1 (habituation session) and 2 (preconditioning test session), the rats were allowed to freely explore the two compartments for $900 \mathrm{~s}$, and the time spent in each compartment during the exploratory period was mea- sured using infrared sensors (Supermex; Muromachi Kikai) positioned on the top cover of each compartment. Rats that spent $>80 \%(>720 \mathrm{~s})$ of the total time ( $900 \mathrm{~s}$ ) on one side on day 2 or showed a difference of $>200$ s in the time spent on one side between days 1 and 2 were excluded from subsequent procedures. Four animals were excluded due to these exclusion criteria. We used a bias-like protocol (Tzschentke, 1998). Specifically, the compartment in which each rat spent less time on day 2 was designated as each animal's drug-paired compartment. The conditioning session was conducted during 6 consecutive days (days 4-9). The animals received bilateral intra-dlBNST injections of NBI27914 (0.5 nmol/ side) and were confined to the drug-paired compartment for $30 \mathrm{~min}$ on days 5,7 , and 9 and received no injections and were confined to the non-drug-paired compartment for $30 \mathrm{~min}$ on days 4,6 , and 8 . On day 11 (postconditioning test session), rats were allowed to freely explore the two compartments for $900 \mathrm{~s}$ and the time spent in each compartment during the exploratory period was measured. The CPP scores were calculated by subtracting the time spent in the drug-paired compartment during the preconditioning test session from the time spent in the same compartment during the postconditioning test session.

Placements of microdialysis probes and injection cannulae. After the in vivo microdialysis and behavioral experiments, the placements of microdialysis probes and injection cannulae were confirmed. Under anesthesia, rats received intra-dlBNST injections of Evans blue $(25 \mu \mathrm{g} / 0.5 \mu \mathrm{l})$ and were decapitated, and their brains were rapidly removed and frozen in powdered dry ice. Coronal sections $(50 \mu \mathrm{m})$ were prepared using a cryostat, thaw-mounted onto slides, stained with thionine, and examined under a microscope $(40 \times)$. Data from rats with misplaced microdialysis probes or misplaced intra-dlBNST injections were excluded from the statistical analyses. Eight animals were excluded due to these exclusion criteria.

Experimental design and statistical analysis. Statistical analyses were performed using GraphPad Prism version 6.00. Differences with $p<0.05$ were considered significant.

In the electrophysiological experiments, 103 rats were assigned to the SNL or sham-operated group. During the course of the experiment, 35 animals were excluded due to the exclusion criteria described above resulting in the final group sizes of the sham-operated $(n=32)$ and SNL $(n=36)$ groups. To compare the sIPSCs between SNL and shamoperated groups 2 weeks after surgery, the data from 5 and 7 cells of sham-operated $(n=3)$ and SNL $(n=3)$ rats, respectively, were analyzed using an unpaired $t$ test. To compare the sIPSCs between SNL and shamoperated groups 4 weeks after surgery, the data from 28 and 25 cells of sham-operated $(n=23)$ and SNL $(n=22)$ rats, respectively, were analyzed using an unpaired $t$ test. These cells from the animals 4 weeks after the surgery were subsequently used to examine the effects of CRF or NBI27914. To examine the effect of CRF on sIPSCs, the data from 13 and 11 cells of sham-operated $(n=12)$ and SNL $(n=10)$ animals, respectively, were analyzed. To examine the effect of NBI27914 on sIPSCs, the data from 15 and 14 cells of sham-operated $(n=11)$ and SNL $(n=13)$ animals, respectively, were analyzed. The frequency and amplitude values were compared before and after the CRF or NBI27914 application using a paired $t$ test within each group. In the electrophysiological recordings in cell-attached mode, firing rates in 13 and 16 cells of shamoperated $(n=6)$ and SNL $(n=11)$ rats, respectively, were analyzed using a Mann-Whitney test. Ten of 16 cells of SNL rats was used to examine the effect of NBI27914 on firing rates. The firing rates were compared before and after the NBI27914 application using a Wilcoxon matched-pairs signed-rank test.

In the FISH experiments assessing CRF mRNA expression, 17 rats were assigned to the SNL or sham-operated group. During the course of the experiment, three (dlBNST) or five (CeA) animals were excluded based on the exclusion criteria for the neuropathic pain model or due to severe tissue damage during the FISH procedure, resulting in the final group sizes of the sham-operated (dlBNST: $n=7$ and CeA: $n=6$ ) and SNL (dlBNST: $n=7$ and CeA: $n=6$ ) groups. Two sections were prepared from the dlBNST of each animal at a level of -0.12 and $-0.24 \mathrm{~mm}$ from bregma. Similarly, two sections were prepared from the CeA of each animal at a level of -2.28 and $-2.52 \mathrm{~mm}$ from bregma. The numbers of CRF mRNA-positive and DAPI-positive neurons on the left and right 
A

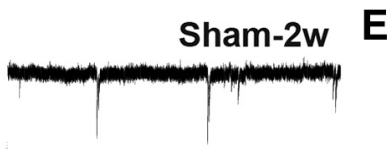

SNL-2w

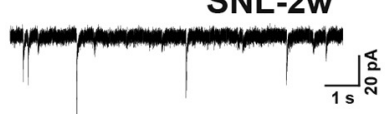

B

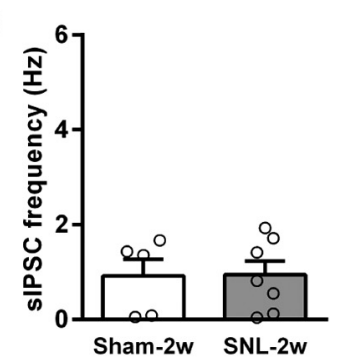

C
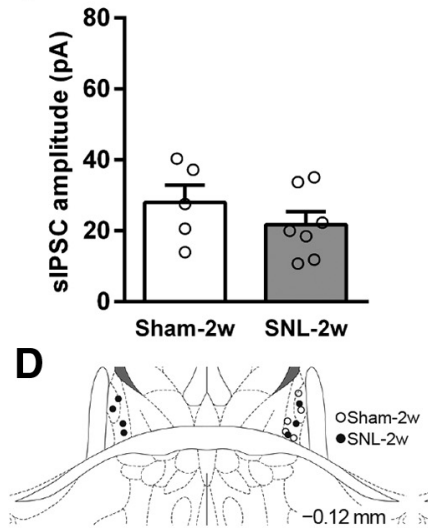

$\mathbf{F}$

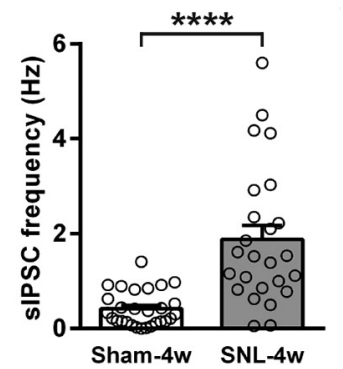

G
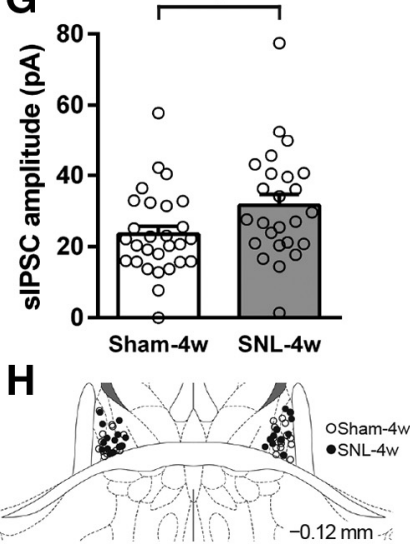

Figure 1. Chronic pain increased the inhibitory inputs to VTA-projecting dIBNST type III neurons. Representative traces of SIPSC recordings in the sham and SNL groups 2 weeks $(\boldsymbol{A})$ and 4 weeks $(\boldsymbol{E})$ after surgery. Frequency $(\boldsymbol{B}, \boldsymbol{F})$ and amplitude $(\boldsymbol{C}, \boldsymbol{G})$ of sIPSCs in the sham and SNL groups 2 weeks $(\boldsymbol{B}, \boldsymbol{C})$ and 4 weeks $(\boldsymbol{F}, \boldsymbol{G})$ after surgery. Data are expressed as means \pm SEM and open circles indicate individual data points [ $\operatorname{sham}(2 \mathrm{w}): n=5$ cells, SNL $(2 \mathrm{w}): n=7$ cells, sham (4w): $n=28$ cells, SNL (4w): $n=25$ cells]. ${ }^{*} p<0.05,{ }^{* * *} p<0.0001$ (unpaired $t$ test). The sites of the recorded BNST neurons $(\boldsymbol{D}, \boldsymbol{H})$. The illustrations of coronal sections are adapted from the atlas of Paxinos and Watson (2007); $-0.12 \mathrm{~mm}$ indicates distance from bregma.

sides of each section were counted to calculate the percentage of CRF mRNA-positive neurons. Unpaired $t$ tests were conducted to compare the percentages of CRF mRNA-positive neurons between the SNL and sham groups.

In the FISH experiments assessing c-fos mRNA expression, 12 rats were assigned to the SNL or sham-operated group. During the course of the experiment, four animals were excluded based on the exclusion criteria for the neuropathic pain model or the intra-dlBNST injection, resulting in the final group sizes of the sham-operated $(n=4)$ and SNL $(n=4)$ groups. For the c-fos mRNA expression analyses, three sections were prepared from the VTA of each animal at a level of $-5.04,-5.28$, and $-5.52 \mathrm{~mm}$ from bregma. Then the numbers of TH-positive and double-positive ( $\mathrm{TH}$ - and c-fos-positive) cells on the left and right sides of each section were counted to calculate the percentage of doublepositive neurons among the total TH-positive neurons. Unpaired $t$ tests were used to compare the percentages of double-positive neurons between the SNL and sham groups.

In the in vivo microdialysis experiments, 44 rats were assigned to the SNL or sham-operated group. During the course of the experiment, 18 animals were excluded due to the exclusion criteria described above resulting in the final group sizes of the sham-operated $(n=14)$ and SNL $(n=12)$ groups. The data of the time courses of extracellular dopamine
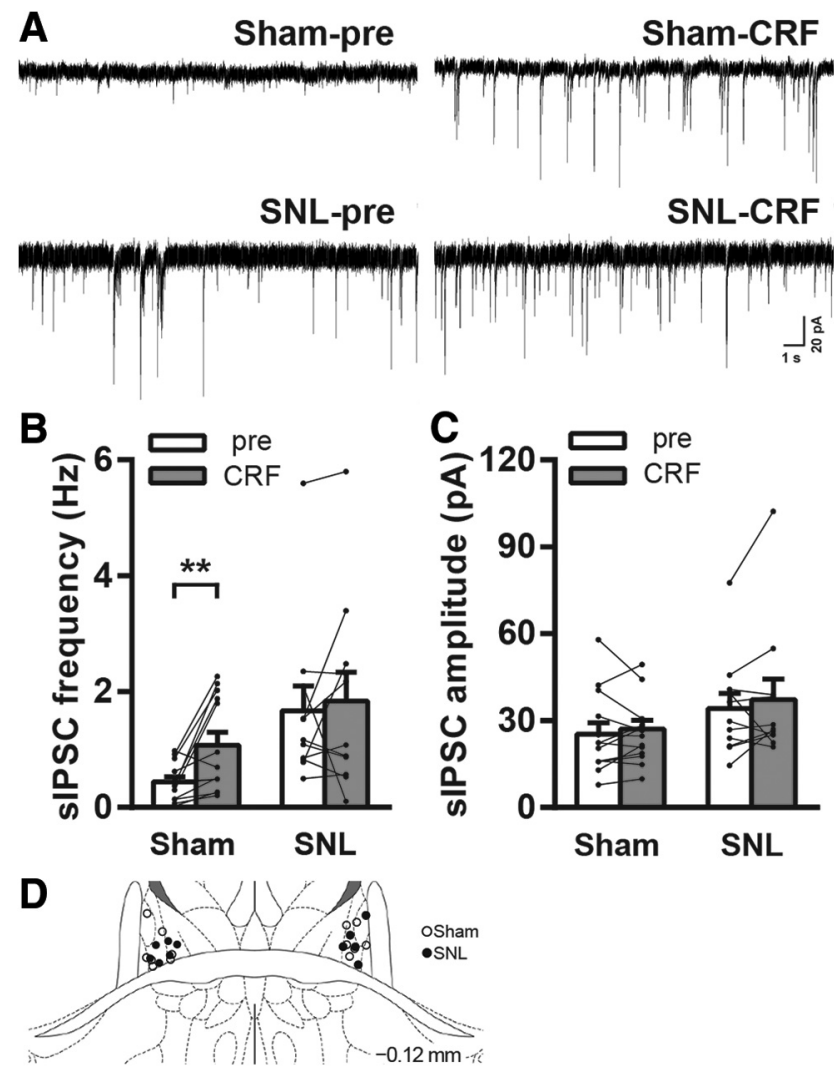

Figure 2. CRF increased the inhibitory inputs to VTA-projecting dIBNST type III neurons in the sham, but not the SNL group 4 weeks after the surgery. Representative traces of sIPSC recordings in the sham and SNL groups $(\boldsymbol{A})$. Frequency $(\boldsymbol{B})$ and amplitude $(\boldsymbol{C})$ of sIPSCs at the time points $0-3$ min before (pre) and 12-15 min after (CRF) the start of CRF application ( $1 \mu \mathrm{M}$, $2 \mathrm{~min}$ ) in the sham and SNL groups (sham: $n=13$ cells, SNL: $n=11$ cells). Data are expressed as means \pm SEM and dots and lines indicate the data recorded from individual neurons before and after (RF application. ${ }^{* *} p<0.01$ (paired $t$ test). The sites of the recorded BNST neurons (D). The illustration of coronal section is adapted from the atlas of Paxinos and Watson (2007) $-0.12 \mathrm{~mm}$ indicates distance from bregma.

levels of the SNL and sham-operated groups were analyzed using a twoway repeated-measures ANOVA.

In the behavioral experiments using a CPP test, 43 rats were assigned to the SNL or sham-operated group. During the course of the experiment, 11 animals were excluded due to the exclusion criteria described above resulting in the final group sizes of the sham-operated $(n=17)$ and SNL $(n=15)$ groups. A paired $t$ test was used to compare the time spent in the drug-paired compartment between the preconditioning and postconditioning test sessions within each group. An unpaired $t$ test was used to compare the CPP scores between the SNL and sham groups.

\section{Results}

sIPSCs increase in VTA-projecting dIBNST neurons of the chronic pain model rats

We previously reported that $\sim 80 \%$ of the VTA-projecting dlBNST neurons are type III (Yamauchi et al., 2018). Thus, sIPSCs were recorded from the VTA-projecting dlBNST type III neurons 2 and 4 weeks after the SNL or sham surgery (Fig. 1). At 2 weeks after the surgery, no difference was observed in the frequency (sham: $0.92 \pm 0.35 \mathrm{~Hz}, n=5$, vs SNL: $0.94 \pm 0.29 \mathrm{~Hz}, n=$ $7, t_{10}=0.049, p=0.96$, unpaired $t$ test; Fig. $\left.1 B\right)$ and amplitude (sham: $28.0 \pm 4.9 \mathrm{pA}, n=5$, vs SNL: $21.8 \pm 3.6 \mathrm{pA}, n=7, t_{10}=$ $1.039, p=0.32$, unpaired $t$ test; Fig. 1C) of sIPSCs between the sham-operated control and SNL groups. By contrast, at 4 weeks after the surgery, the frequency of sIPSCs in the VTA-projecting dlBNST type III neurons of the SNL group was significantly 
A
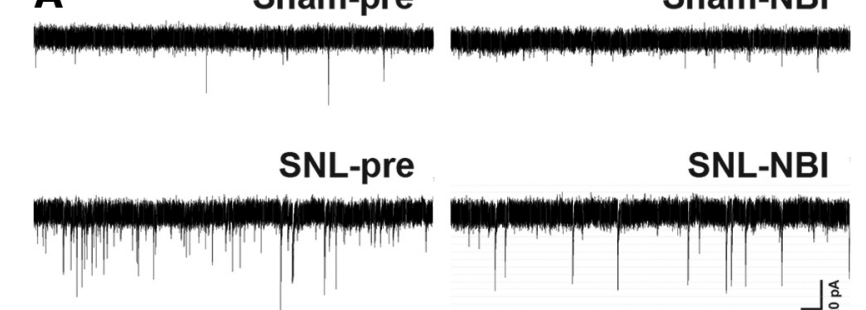

B
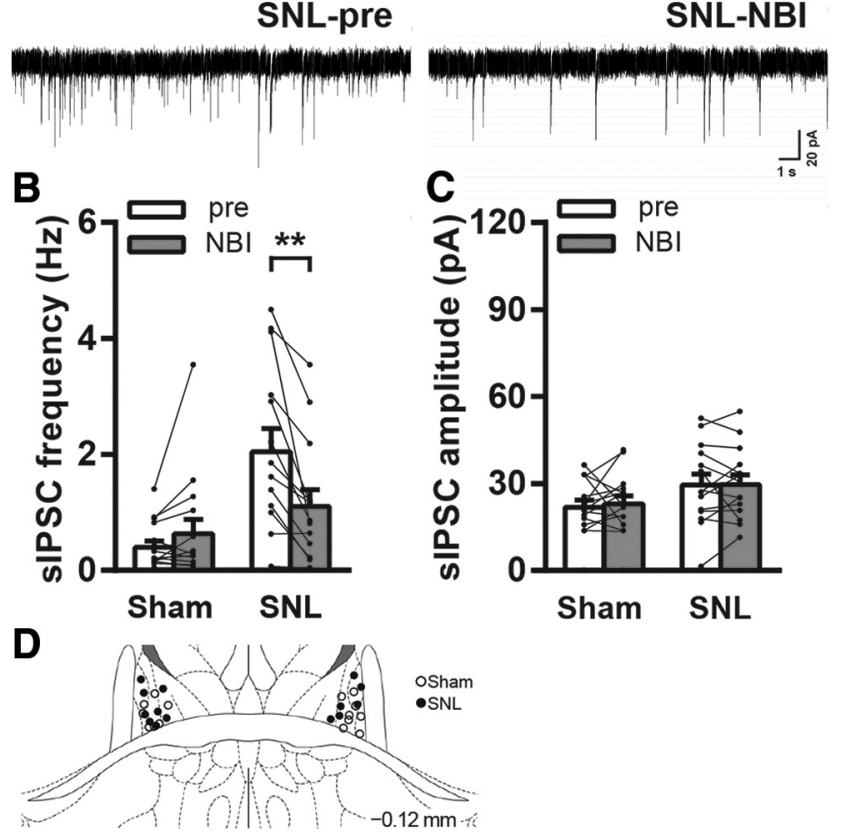

Figure 3. NBI27914 decreased the inhibitory inputs to VTA-projecting dIBNST type III neurons in the SNL, but not the sham group 4 weeks after the surgery. Representative traces of sIPSC recordings in the sham and SNL groups $(\boldsymbol{A})$. Frequency $(\boldsymbol{B})$ and amplitude $(\boldsymbol{C})$ of sIPSCs at the time points $0-3 \mathrm{~min}$ before (pre) and 12-15 min after (NBI) the start of NBI27914 application ( $1 \mu \mathrm{M}, 15 \mathrm{~min}$ ) in the sham and SNL groups (sham: $n=15$ cells, SNL: $n=14$ cells). Data are expressed as means \pm SEM and dots and lines indicate the data recorded from individual neurons before and after NBI27914 application. ${ }^{* *} p<0.01$ (paired $t$ test). The sites of the recorded BNST neurons $(\boldsymbol{D})$. The illustration of coronal section is adapted from the atlas of Paxinos and Watson (2007); $-0.12 \mathrm{~mm}$ indicates distance from bregma.

higher than that of the sham group (sham: $0.42 \pm 0.07 \mathrm{~Hz}, n=28$ vs SNL: $1.88 \pm 0.29 \mathrm{~Hz}, n=25, t_{51}=5.165, p<0.0001$, unpaired $t$ test; Fig. $1 F$ ). The amplitude of sIPSCs also increased in the SNL group (sham: $23.5 \pm 2.2 \mathrm{pA}, n=28$, vs SNL: $31.6 \pm 3.1 \mathrm{pA}, n=$ $25, t_{51}=2.169, p=0.035$, unpaired $t$ test; Fig. $\left.1 G\right)$.

Effects of CRF on sIPSCs in VTA-projecting dlBNST neurons of chronic pain model rats

The effects of CRF on the sIPSCs in the VTA-projecting dlBNST type III neurons were examined 4 weeks after the SNL or sham surgery (Fig. 2). In the sham group, a bath application of $1 \mu \mathrm{M}$ CRF significantly increased the frequency of sIPSCs (pre: $0.44 \pm$ $0.09 \mathrm{~Hz}$, vs CRF: $1.07 \pm 0.22 \mathrm{~Hz}, n=13, t_{12}=3.273, p=0.0067$, paired $t$ test; Fig. $2 B$ ). The amplitude of sIPSCs was not different before and after the CRF application (pre: $25.4 \pm 3.9$ pA, vs CRF: $25.7 \pm 3.1 \mathrm{pA}, n=13, t_{12}=0.159, p=0.88$, paired $t$ test; Fig. $2 C$ ). In the SNL group, CRF did not affect the frequency of sIPSCs (pre: $1.66 \pm 0.43 \mathrm{~Hz}$, vs CRF: $1.84 \pm 0.50 \mathrm{~Hz}, n=11, t_{10}=0.548$, $p=0.60$, paired $t$ test; Fig. $2 B$ ), and the amplitude of sIPSCs was not different before and after the CRF application (pre: $34.3 \pm 5.2$ pA, vs CRF: $37.2 \pm 7.1 \mathrm{pA}, n=11, t_{10}=0.916, p=0.38$, paired $t$ test; Fig. 2C).

Effects of NBI27914 on sIPSCs in VTA-projecting dlBNST neurons of chronic pain model rats

The effects of NBI27914, a CRF type 1 receptor antagonist, on sIPSCs in the VTA-projecting dlBNST type III neurons were ex-
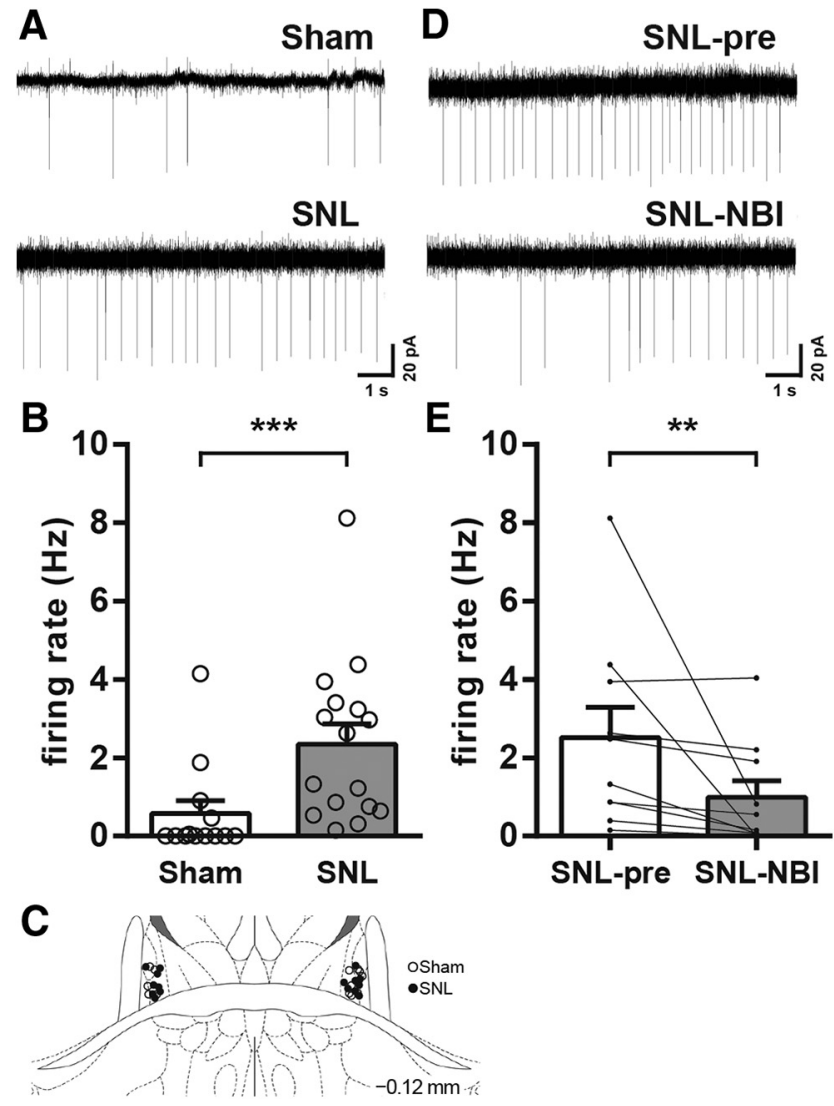

Figure 4. Chronic pain increased the firing rates of dIBNST type II neurons. Representative traces obtained from cell-attached recordings of the sham and SNL groups $(\boldsymbol{A})$. Firing rates in the sham and SNL groups $(\boldsymbol{B})$. Data are expressed as means \pm SEM and open circles indicate individual data points (sham: $n=13$ cells, SNL: $n=16$ cells). ${ }^{* * *} p<0.001$ (Mann-Whitney test). The sites of the recorded BNST neurons $(\boldsymbol{C})$. The illustration of coronal section is adapted from the atlas of Paxinos and Watson (2007); $-0.12 \mathrm{~mm}$ indicates distance from bregma. Representative traces of the firing rates of dIBNST type II neurons of the SNL rats before and after NBI27914 application ( $\boldsymbol{D})$. Firing rates at the time points $0-3 \mathrm{~min}$ before (pre) and 12-15 min after (NBI) the start of NBI27914 application (1 $\mu \mathrm{M}, 15 \mathrm{~min}$ ). Data are expressed as means \pm SEM and dots and lines indicate the data recorded from individual neurons before and after NBI27914 application (SNL: $n=10$ cells). ${ }^{* *} p<0.01$ (Wilcoxon matched-pairs signed-rank test).

amined 4 weeks after the SNL or sham surgery (Fig. 3). In the sham group, a bath application of $1 \mu \mathrm{M}$ NBI27914 did not affect the frequency (pre: $0.40 \pm 0.11 \mathrm{~Hz}$ vs NBI27914: $0.63 \pm 0.24 \mathrm{~Hz}$, $n=15, t_{14}=1.560, p=0.14$, paired $t$ test; Fig. $3 B$ ) or amplitude (pre: $21.8 \pm 2.4 \mathrm{pA}$ vs NBI27914: $23.0 \pm 2.7 \mathrm{pA}, n=15, t_{14}=$ $0.495, p=0.63$, paired $t$ test; Fig. $3 C$ ) of the sIPSCs. In the SNL group, a bath application of NBI27914 significantly decreased the frequency of sIPSCs (pre: $2.05 \pm 0.40 \mathrm{~Hz}$ vs NBI27914: $1.10 \pm$ $0.29 \mathrm{~Hz}, n=14, t_{13}=4.042, p=0.0014$, paired $t$ test; Fig. $3 B$ ). The amplitude of the sIPSCs was not different before and after the NBI27914 application (pre: $29.6 \pm 3.8$ pA vs NBI27914: $29.8 \pm$ $3.3 \mathrm{pA}, n=14, t_{13}=0.102, p=0.92$, paired $t$ test; Fig. $3 C$ ).

Excitability of dlBNST type II neurons increases in chronic pain model rats

Hammack et al. (2007) raised the possibility that some portion of type II dlBNST neurons may be local-circuit GABAergic interneurons, and that type II neurons should make synaptic contact with other type(s) of dlBNST neurons to regulate dlBNST output. Therefore, the increase in the inhibitory inputs to the VTA-projecting dlBNST type III neurons observed in the SNL 
A

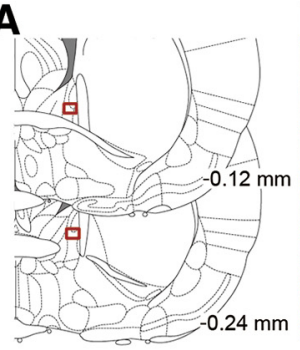

D

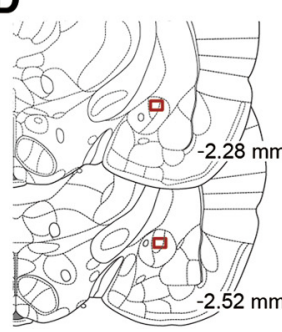

B

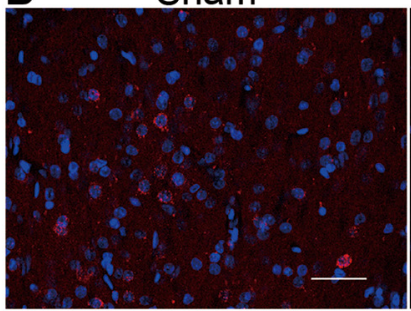

E

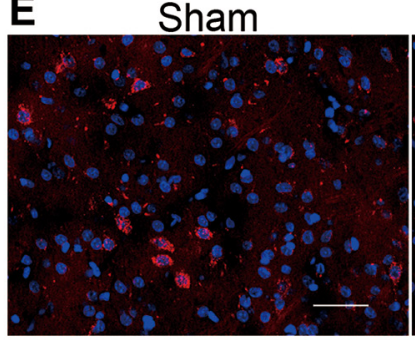

SNL
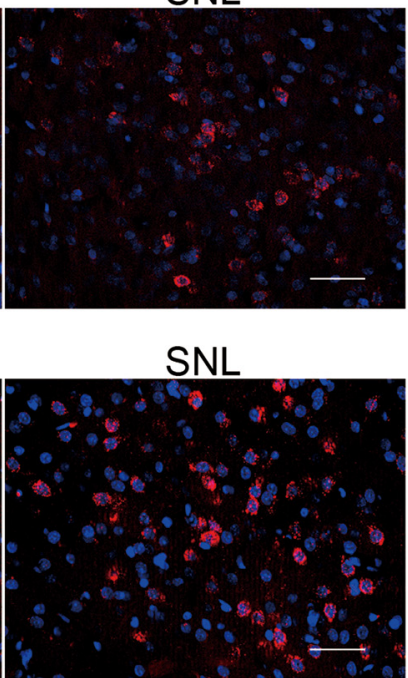

C

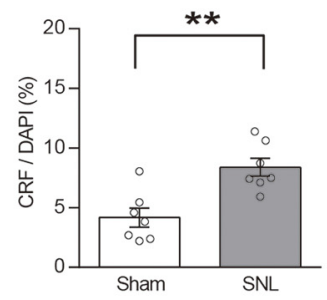

F

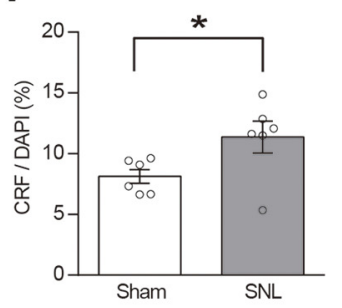

Figure 5. Chronic pain increased CRF mRNA expression in the extended amygdala. Placements of the ROIs (red square) for the analyses of CRF mRNA expression in the dIBNST ( $\boldsymbol{A}$ ) and CeA ( $\boldsymbol{D}$ ). The illustrations of coronal sections are adapted from the atlas of Paxinos and Watson (2007); -0.12 and $-0.24 \mathrm{~mm}$ on the illustrations of the dIBNST (A) and -2.28 and $-2.52 \mathrm{~mm}$ on the illustrations of the CeA $(\boldsymbol{D})$ indicate distances from bregma. Representative images of CRF mRNA expression (red) and DAPI staining (blue) in the dIBNST ( $\boldsymbol{B}$ ) and (eA ( $\boldsymbol{E}$ ) of the sham (left) and SNL (right) rats. Scale bar, $50 \mu \mathrm{m}$. Percentages of CRF mRNA-positive neurons in the dIBNST ( $\boldsymbol{C})$ and CeA $(\boldsymbol{F})$ of the sham and SNL rats; data are expressed as means \pm SEM and open circles indicate individual data points. ${ }^{*} p<0.05,{ }^{* *} p<0.01$ (unpaired $t$ test).

group may be due to enhanced excitation of type II dlBNST neurons. To address this hypothesis, firing rates in dlBNST type II neurons were recorded in cell-attached mode 4 weeks after the SNL or sham surgery (Fig. $4 A$ ). The firing rates of dlBNST type II neurons in the SNL group were significantly higher than those in the sham group (sham: $0.57 \pm 0.33 \mathrm{~Hz}, n=13$ vs SNL: $2.35 \pm$ $0.52 \mathrm{~Hz}, n=16, p=0.0006$, Mann-Whitney test; Fig. $4 B$ ). The increased firing rates observed in the SNL group were significantly suppressed by a bath application of $1 \mu \mathrm{M}$ NBI27914 (pre: $2.52 \pm 0.77 \mathrm{~Hz}$ vs NBI27914: $0.99 \pm 0.42 \mathrm{~Hz}, n=10, t_{9}=2.018$, $p=0.0039$, Wilcoxon matched-pairs signed-rank test; Fig. $4 D, E)$.

\section{CRF mRNA expression in the extended amygdala of chronic pain model rats}

Because the electrophysiological experiments suggested an enhanced CRF signaling within the dlBNST, CRF mRNA expressions in the dlBNST and CeA, which sends dense projections to the BNST, were examined 4 weeks after the SNL or sham surgery (Fig. 5). CRF mRNA expression in the dlBNST increased in the SNL group compared with the sham group (sham: $4.18 \pm 0.8 \%$, $n=7$ vs SNL: $8.39 \pm 0.7 \%, n=7, t_{12}=3.88, p=0.0022$, unpaired $t$ test; Fig. $5 C$ ). Similarly, CRF mRNA expression in the CeA increased in the SNL group compared with the sham group (sham: $8.12 \pm 0.6 \%, n=6$ vs SNL: $11.4 \pm 1.3 \%, n=6, t_{10}=2.28$, $p=0.046$, unpaired $t$ test; Fig. $5 F)$.

Effects of intra-dlBNST injection of NBI27914 on c-fos mRNA expression in the VTA dopaminergic neurons in chronic pain model rats

The electrophysiological experiments showed that NBI27914 reversed the increased inhibitory inputs to VTA-projecting dIBNST neurons in brain slices prepared from the chronic pain rats. To assess the effects of the blockade of intra-dlBNST CRF signaling on the activity of VTA dopaminergic neurons in chronic pain rats, c-fos mRNA expression in VTA TH-positive neurons was examined at 4 weeks after the SNL or sham surgery (Fig. 6).
A
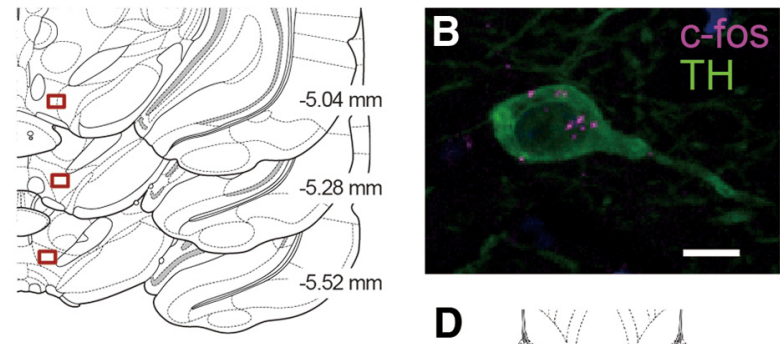

C
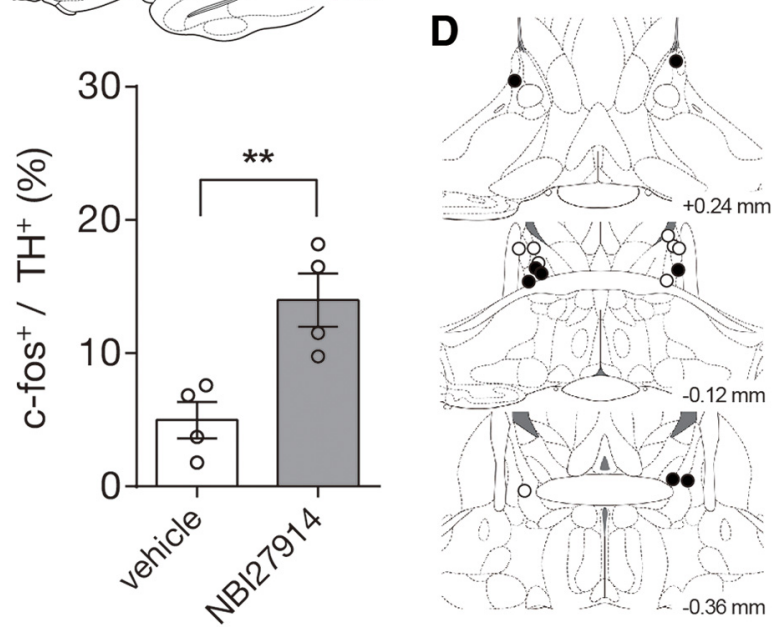

Figure 6. Intra-dIBNST injections of NBI27914 increased c-fos mRNA expression in VTA THpositive neurons in chronic pain model rats. Placements of the ROIs (red square) for the analyses of $c-$ fos mRNA expression in the VTA $(\boldsymbol{A})$. The illustrations of coronal sections are adapted from the atlas of Paxinos and Watson (2007); $-5.04,-5.28$, and $-5.52 \mathrm{~mm}$ indicate distances from bregma. The representative image of a c-fos (magenta)- and TH (green)-positive neuron (B). Scale bar, $10 \mu \mathrm{m}$. Percentages of double-positive $\left(\mathrm{c}-\mathrm{fos}^{+} / \mathrm{TH}^{+}\right)$neurons among the total TH-positive neurons in chronic pain model rats injected with vehicle $(n=4)$ or NBI27914 ( $n=$ 4) into the dIBNST (C). Data are expressed as means \pm SEM and open circles indicate individual data points. ${ }^{* *} p<0.01$ (unpaired $t$ test). Placements of the microinjection cannulae (vehicle: open circle; NBI27914: closed circle, D). Illustrations of coronal sections are adapted from the atlas of Paxinos and Watson (2007); $+0.24,-0.12$ and $-0.36 \mathrm{~mm}$ indicate distances from bregma. 
Bilateral intra-dlBNST injections of NBI27914 significantly increased c-fos mRNA expression in the VTA TH-positive neurons compared with the vehicle-injected group (vehicle: $4.98 \pm 1.4 \%$, $n=4$ vs NBI27914: $14.0 \pm 2.0 \%, n=4, t_{6}=3.73, p=0.0098$, unpaired $t$ test; Fig. $6 C$ ).

\section{Effects of intra-dlBNST injections of NBI27914 on the} mesolimbic reward system in chronic pain model rats To examine the effect of blockade of intra-dlBNST CRF signaling on the mesolimbic reward circuit in the chronic pain model animals, extracellular dopamine levels in the NAc were measured using an in vivo microdialysis technique 4 weeks after the SNL or sham surgery. Intra-dlBNST injections of NBI27914 significantly increased extracellular dopamine levels in the NAc of the SNL rats compared with those of the sham-operated animals (twoway repeated-measures ANOVA: interaction, $F_{(20,480)}=4.20$, $p<0.0001$; Fig. $7 A$ ).

Since blockade of intra-dlBNST CRF signaling increased extracellular dopamine levels in the NAc of the chronic pain model rats, this blocking may produce any rewarding effect. To test this idea, we performed a CPP test 4 weeks after the SNL or sham surgery. In the SNL group, the time spent in the drug-paired compartment after conditioning with the intra-dlBNST injections of NBI27914 (postconditioning test session) increased significantly compared with that before the conditioning (preconditioning test session) (pre: $295.0 \pm 19.5 \mathrm{~s}$ vs post: $486.3 \pm$ $20.3 \mathrm{~s}, n=15, t_{14}=8.153, p<0.0001$, paired $t$ test; Fig. $\left.7 B\right)$. In the sham group, no significant difference was observed in the time spent in the drug-paired compartment between the preconditioning and postconditioning test sessions (pre: $335.1 \pm 9.8 \mathrm{~s}$ vs post: $342.4 \pm 19.9 \mathrm{~s}, n=17, t_{16}=0.4762, p=0.64$, paired $t$ test; Fig. $7 B$ ). The CPP score of the SNL group was significantly higher than that of the sham group (sham: $7.3 \pm 15.3 \mathrm{~s}, n=17$ vs SNL: $191.3 \pm 23.5 \mathrm{~s}, n=15, t_{30}=6.715, p<0.0001$, unpaired $t$ test; Fig. 7C).

\section{Discussion}

We previously reported that CRF selectively depolarizes dIBNST type II neurons (Ide et al., 2013) and increases the inhibitory synaptic inputs to dlBNST type III neurons (Nagano et al., 2015) in naive rats. The present study showed that: (1) the sIPSC frequency in the VTA-projecting dlBNST type III neurons increased during chronic pain, (2) CRF increased the sIPSC frequency in the VTA-projecting dlBNST type III neurons of the shamoperated control, but not the chronic pain rats, suggesting the occlusion of the effect of exogenously applied CRF in the chronic pain group, and (3) NBI27914, a CRF type 1 receptor antagonist, decreased sIPSC frequency in the chronic pain, but not the control group. These results suggest that enhanced CRF signaling tonically increased inhibitory inputs to the VTA-projecting dlBNST type III neurons during chronic pain. Two brain regions are considered possible sources of CRF-containing nerve terminals in the dlBNST: the dlBNST intrinsic neurons (Morin et al., 1999; Silberman et al., 2013; Pomrenze et al., 2015) and the CeA neurons projecting to the dlBNST (Asok et al., 2018). Histological analyses using a FISH technique revealed that CRF mRNA expression increased in the dlBNST and CeA of chronic pain model rats. Similar findings were reported by Rouwette et al. (2012). These authors demonstrated that CRF mRNA expression increased in the dlBNST and CeA of neuropathic pain model rats prepared by chronic constriction injury of the sciatic nerve $24 \mathrm{~d}$ after surgery.
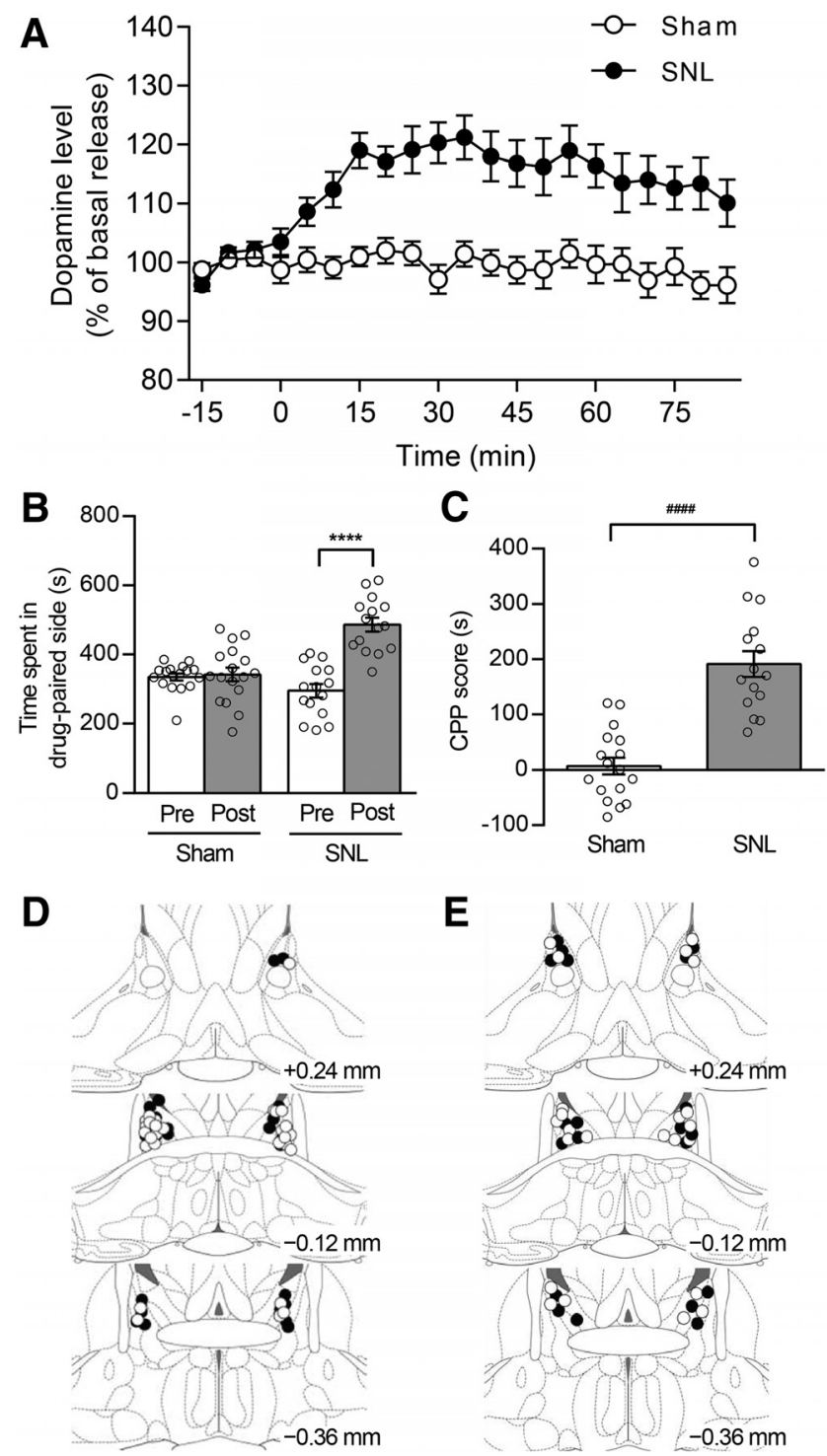

Figure 7. Intra-dIBNST injections of NBI27914 induced dopamine release in the NAc of the chronic pain model rats. Time courses of extracellular dopamine levels in the NAc of the sham (open circle, $n=14$ ) and SNL (closed circle, $n=12$ ) groups $(\boldsymbol{A})$. NBI27914 was bilaterally injected into the dIBNST at time 0 . Data are expressed as means \pm SEM. Intra-dIBNST injections of NBI27914 induced CPP in the chronic pain model rats ( $\boldsymbol{B}, \boldsymbol{C}$. Time spent in the drug-paired compartment during the preconditioning (Pre) and postconditioning (Post) test sessions in the sham and SNL groups $(\boldsymbol{B})$. The CPP scores were calculated by subtracting the time spent in the drug-paired compartment during the preconditioning test session from the time spent in the same compartment during the postconditioning test session (C). Data are expressed as means \pm SEM and open circles indicate individual data points (sham: $n=17$, SNL: $n=15$ ).

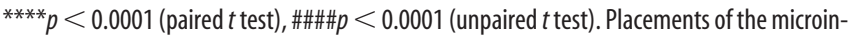
jection cannulae (sham: open circle; SNL: closed circle) in the in vivo microdialysis experiments $(\boldsymbol{D})$ and the (PP tests $(\boldsymbol{E})$. The illustrations of coronal sections are adapted from the atlas of Paxinos and Watson (2007); $+0.24,-0.12$ and $-0.36 \mathrm{~mm}$ indicate distances from bregma.

Hammack et al. (2007) suggested that some portion of dlBNST type II neurons may be local-circuit GABAergic interneurons and that type II neurons should make synaptic contact with other type(s) of dlBNST neurons to regulate output of the dlBNST. Therefore, excitation of dlBNST type II neurons is expected to increase the inhibitory synaptic transmission to other type(s) of dlBNST neurons. Thus, we hypothesized that the increased inhibitory inputs to VTA-projecting dlBNST neurons are due to increased excitability of the dlBNST type II neurons. Elec- 
trophysiological recordings in cell-attached mode revealed that the firing rates in dlBNST type II neurons of the chronic pain rats were significantly higher than those of the sham-operated control animals, indicating elevated excitability of the dlBNST type II neurons during chronic pain. Furthermore, the elevated excitability in dlBNST type II neurons was suppressed by a bath application of the CRF type 1 receptor antagonist NBI27914. These findings suggest that enhanced CRF signaling may elevate the excitability of dlBNST type II neurons, thereby increasing the inhibitory inputs to VTA-projecting dlBNST type III neurons.

NBI27914 was injected into the dlBNST to reverse the increased inhibitory inputs to the VTA-projecting dlBNST neurons and then its effects on VTA dopaminergic neuron activity were examined. Histochemical analyses revealed that bilateral intradlBNST injections of NBI27914 increased c-fos mRNA expression in TH-positive cells, which suggests that the activation of VTA-projecting dlBNST neurons increased dopaminergic neuron activity. This result is consistent with those of Georges and Aston-Jones (2001), who demonstrated the excitatory control of VTA dopaminergic neurons by VTA-projecting BNST neurons using an in vivo electrophysiological technique. Recently, Kaufling et al. (2017) identified direct monosynaptic connections between BNST neurons and VTA dopaminergic neurons in mice and rats. These authors demonstrated that the inhibition of VTA dopaminergic neurons is the main response to electrical stimulation of the BNST ( $84.6 \%$ and $62.5 \%$ of responding neurons were inhibited in mice and rats, respectively) whereas $15.4 \%$ and $37.5 \%$ of responding neurons are excited by BNST electrical stimulation in mice and rats, respectively. These findings appear to be inconsistent with the present results showing that the activation of VTA-projecting dlBNST neurons increased c-fos mRNA expression in VTA TH-positive neurons as well as those of previous optogenetic studies showing that photoactivation of VTA-projecting BNST neurons induces reward-related behaviors in a real-time place preference test (Jennings et al., 2013; Kim et al., 2013), which suggests that VTA-projecting BNST neurons positively regulate the mesolimbic reward circuit. This inconsistency could be explained by the presence of indirect disynaptic connections between BNST neurons and VTA dopaminergic neurons. More specifically, most VTA-projecting BNST neurons are GABAergic neurons that preferentially form synapses on VTA GABAergic neurons (Kudo et al., 2012). Thus, the activation of VTA-projecting BNST neurons should promote VTA dopaminergic neuron activity through the inhibition of VTA GABAergic neurons that negatively regulate VTA dopaminergic neurons. This disinhibition mechanism may be important for the regulation of VTA dopaminergic neurons via VTA-projecting BNST neurons.

We previously reported that sucrose solution intake as a reward induces intra-NAc dopamine release in the SNL and sham groups during the early phase of neuropathic pain (2 weeks after the surgery), while it induces intra-NAc dopamine release in the sham, but not the SNL, group during the late phase of neuropathic pain (4 weeks after the surgery) (Kato et al., 2016). In addition, Dellarole et al. (2014) reported that the anhedonic behavior in a sucrose preference test starts 4 weeks after surgery in neuropathic pain model mice. These findings suggest that dysfunction of the mesolimbic reward circuit develops in a timedependent manner. Consistent with these findings, the present study showed that inhibitory inputs to the VTA-projecting dlBNST neurons increased at 4 weeks, but not at 2 weeks, following SNL surgery, suggesting the involvement of increased inhibitory inputs to the VTA-projecting dIBNST neurons in the dysfunction of the mesolimbic reward circuit during chronic pain. It is possible that tonic inhibition of VTA-projecting dlBNST neurons may cause the dysfunction of the mesolimbic reward circuit observed in the late phase of neuropathic pain. To address this issue, we injected NBI27914, a CRF type 1 receptor antagonist, into the dlBNST to reverse the increased inhibitory inputs to the VTA-projecting dlBNST neurons and examined its effects on the mesolimbic reward system using an in vivo microdialysis technique and a CPP test. In vivo microdialysis revealed that bilateral intra-dlBNST injections of NBI27914 increased intra-NAc dopamine release in the SNL, but not the sham group. Furthermore, bilateral intra-dlBNST injections of NBI27914 induced CPP in the SNL, but not the sham group. These results suggest that dysfunction of the mesolimbic reward circuit during chronic pain is at least, in part, due to the enhanced CRF signaling in the dlBNST, which increases inhibitory inputs to the VTAprojecting dlBNST neurons.

In conclusion, this is the first report demonstrating chronic pain-induced neuroplastic change in the inhibitory inputs to the VTA-projecting dlBNST neurons, which is mediated by enhanced CRF signaling within the dIBNST. This neuroplastic change leads to tonic suppression of the mesolimbic dopaminergic system, which may be involved in depressive mood and anhedonia under the chronic pain condition.

\section{References}

Alheid GF, Heimer L (1988) New perspectives in basal forebrain organization of special relevance for neuropsychiatric disorders: the striatopallidal, amygdaloid, and corticopetal components of substantia innominate, Neuroscience 27:1-39.

Asok A, Draper A, Hoffman AF, Schulkin J, Lupica CR, Rosen JB (2018) Optogenetic silencing of a corticotropin-releasing factor pathway from the central amygdala to the bed nucleus of the stria terminalis disrupts sustained fear. Mol Psychiatry 23:914-922.

Bair MJ, Robinson RL, Katon W, Kroenke K (2003) Depression and pain comorbidity. Arch Intern Med 163:2433-2445.

Chaplan SR, Bach FW, Pogrel JW, Chung JM, Yaksh TL (1994) Quantitative assessment of tactile allodynia in the rat paw. J Neurosci Methods 53:5563.

Davis M, Walker DL, Miles L, Grillon C (2010) Phasic vs sustained fear in rats and humans: role of the extended amygdala in fear vs anxiety. Neuropsychopharmacology 35:105-135.

Dellarole A, Morton P, Brambilla R, Walters W, Summers S, Bernardes D, Grilli M, Bethea JR (2014) Neuropathic pain-induced depressive-like behavior and hippocampal neurogenesis and plasticity are dependent on TNFR1 signaling, Brain Behav Immun 41:65-81.

Doan L, Manders T, Wang J (2015) Neuroplasticity underlying the comorbidity of pain and depression. Neural Plast 2015:504691.

Georges F, Aston-Jones G (2001) Potent regulation of midbrain dopamine neurons by the bed nucleus of the stria terminalis. J Neurosci 21:RC160.

Hammack SE, Mania I, Rainnie DG (2007) Differential expression of intrinsic membrane currents in defined cell types of the anterolateral bed nucleus of the stria terminalis. J Neurophysiol 98:638-656.

Ide S, Hara T, Ohno A, Tamano R, Koseki K, Naka T, Maruyama C, Kaneda K, Yoshioka M, Minami M (2013) Opposing roles of corticotropinreleasing factor and neuropeptide $Y$ within the dorsolateral bed nucleus of the stria terminalis in the negative affective component of pain in rats. J Neurosci 33:5881-5894.

Jennings JH, Sparta DR, Stamatakis AM, Ung RL, Pleil KE, Kash TL, Stuber GD (2013) Distinct extended amygdala circuits for divergent motivational states. Nature 496:224-228.

Kato T, Ide S, Minami M (2016) Pain relief induces dopamine release in the rat nucleus accumbens during the early but not late phase of neuropathic pain. Neurosci Lett 629:73-78.

Kaufling J, Girard D, Maitre M, Leste-Lasserre T, Georges F (2017) Speciesspecific diversity in the anatomical and physiological organisation of the BNST-VTA pathway. Eur J Neurosci 45:1230-1240.

Kim SY, Adhikari A, Lee SY, Marshel JH, Kim CK, Mallory CS, Lo M, Pak S, Mattis J, Lim BK, Malenka RC, Warden MR, Neve R, Tye KM, Deisseroth 
K (2013) Diverging neural pathways assemble a behavioural state from separable features in anxiety. Nature 496:219-223.

Kono J, Konno K, Talukder AH, Fuse T, Abe M, Uchida K, Horio S, Sakimura K, Watanabe M, Itoi K (2017) Distribution of corticotropin-releasing factor neurons in the mouse brain: a study using corticotropin-releasing factor-modified yellow fluorescent protein knock-in mouse. Brain Struct Funct 222:1705-1732.

Kudo T, Uchigashima M, Miyazaki T, Konno K, Yamasaki M, Yanagawa Y, Minami M, Watanabe M (2012) Three types of neurochemical projection from the bed nucleus of the stria terminalis to the ventral tegmental area in adult mice. J Neurosci 32:18035-18046.

Li Y, Dorsi MJ, Meyer RA, Belzberg AJ (2000) Mechanical hyperalgesia after an L5 spinal nerve lesion in the rat is not dependent on input from injured nerve fibers. Pain 85:493-502.

Minami M (2019) The role of the bed nucleus of the stria terminalis in pain-induced aversive motivation. Curr Opin Behav Sci 26:46-53.

Minami S, Satoyoshi H, Ide S, Inoue T, Yoshioka M, Minami M (2017) Suppression of reward-induced dopamine release in the nucleus accumbens in animal models of depression: Differential responses to drug treatment. Neurosci Lett 650:72-76.

Morin SM, Ling N, Liu XJ, Kahl SD, Gehlert DR (1999) Differential distribution of urocortin- and corticotropin-releasing factor-like immunoreactivities in the rat brain. Neuroscience 92:281-291.

Nagano Y, Kaneda K, Maruyama C, Ide S, Kato F, Minami M (2015) Corticotropin-releasing factor enhances inhibitory synaptic transmission to type III neurons in the bed nucleus of the stria terminalis. Neurosci Lett 600:56-61.

Nestler EJ, Carlezon WA Jr (2006) The mesolimbic dopamine reward circuit in depression. Biol Psychiat 59:1151-1159.

Ozaki S, Narita M, Ino M, Sugita J, Matsumura Y, Suzuki T (2002) Suppression of the morphine-induced rewarding effect in the rat with neuropathic pain: implication of the reduction in mu-opioid receptor functions in the ventral tegmental area. J Neurochem 82:1192-1198.
Paxinos G, Watson C (2007) The rat brain in stereotaxic coordinates, Ed 6. San Diego: Academic.

Pomrenze MB, Millan EZ, Hopf FW, Keiflin R, Maiya R, Blasio A, Dadgar J, Kharazia V, De Guglielmo G, Crawford E, Janak PH, George O, Rice KC, Messing RO (2015) A transgenic rat for investigating the anatomy and function of corticotrophin releasing factor circuits. Front Neurosci 9:487.

Rouwette T, Vanelderen P, de Reus M, Loohuis NO, Giele J, van Egmond J, Scheenen W, Scheffer GJ, Roubos E, Vissers K, Kozicz T (2012) Experimental neuropathy increases limbic forebrain CRF. Eur J Pain 16:61-71.

Russo SJ, Nestler EJ (2013) The brain reward circuitry in mood disorders. Nat Rev Neurosci 14:609-625.

Shinohara F, Kihara Y, Ide S, Minami M, Kaneda K (2014) Critical role of cholinergic transmission from the laterodorsal tegmental nucleus to the ventral tegmental area in cocaine-induced place preference. Neuropharmacology 79:573-579.

Silberman Y, Matthews RT, Winder DG (2013) A corticotropin releasing factor pathway for ethanol regulation of the ventral tegmental area in the bed nucleus of the stria terminalis. J Neurosci 33:950-960.

Taylor AM, Castonguay A, Taylor AJ, Murphy NP, Ghogha A, Cook C, Xue L, Olmstead MC, De Koninck Y, Evans CJ, Cahill CM (2015) Microglia disrupt mesolimbic reward circuitry in chronic pain. J Neurosci 35: $8442-8450$.

Tzschentke TM (1998) Measuring reward with the conditioned place preference paradigm: a comprehensive review of drug effects, recent progress and new issues. Prog Neurobiol 56:613-672.

Yalcin I, Barthas F, Barrot M (2014) Emotional consequences of neuropathic pain: insight from preclinical studies. Neurosci Biobehav Rev 47:154-164.

Yamauchi N, Takahashi D, Sugimura YK, Kato F, Amano T, Minami M (2018) Activation of the neural pathway from the dorsolateral bed nucleus of the stria terminalis to the central amygdala induces anxiety-like behaviors. Eur J Neurosci 48:3052-3061. 